\title{
Automatic Extraction of Hierarchical Urban Networks: A Micro-Spatial Approach
}

\author{
Rui Carvalho ${ }^{1}$ and Michael Batty ${ }^{2}$ \\ 1 The Bartlett School of Graduate Studies \\ rui.carvalho@ucl.ac.uk, \\ 2 Centre for Advanced Spatial Analysis \\ University College London, \\ 1-19 Torrington Place, London WC1E 6BT, UK \\ m. batty@ucl.ac.uk
}

\begin{abstract}
We present an image processing technique for the identification of 'axial lines' [1] from ridges in isovist fields first proposed by Rana [23. These ridges are formed from the maximum diametric lengths of the individual isovists, sometimes called viewsheds, that make up the isovist fields [4]. We discuss current strengths and weaknesses of the method, and show how it can be implemented easily and effectively.
\end{abstract}

\section{Axial Maps as Skeletons for Urban Morphology}

Axial lines are used in 'space syntax' to simplify connections between spaces that make up an urban or architectural morphology. Usually they are defined manually by partitioning the space into the smallest number of largest convex subdivisions and defining these lines as those that link these spaces together. Subsequent analysis of the resulting set of lines (which is called an 'axial map') enables the relative nearness or accessibility of these lines to be computed. These can then form the basis for ranking the relative importance of the underlying spatial subdivisions and associating this with measures of urban intensity, density, or traffic flow [156]. Progress has been slow at generating these lines automatically. Lack of agreement on their definition and lack of awareness as to how similar problems have been treated in fields such as pattern recognition, robotics and computer vision have inhibited explorations of the problem and only very recently have there been any attempts to evolve methods for the automated generation of such lines [7/8]4.

One obvious advantage of a rigorous algorithmic definition of axial lines is the potential use of the computer to free humans from the tedious tracing of lines on large urban systems. Perhaps less obvious is the insight that mathematical procedures may bring about urban networks, and their context in the burgeoning body of research into the structure and function of complex networks 9010. Indeed, on one hand urban morphologies display a surprising degree of universality [11 12]131415], but little is yet known about the relation between this observed universality and the transport and social networks embedded within 
urban space (but see [16,17]). On the other hand, axial maps are a substrate for human navigation and rigorous extraction of axial lines may substantiate the development of models for processes that take place on urban networks which range from issues covering the efficiency of navigation, and the vulnerability of network nodes and links to failure, attack and related crises.

Axial maps can be regarded as members of a larger family of axial representations (often called skeletons) of 2D images. There is a vast literature on this, originating with the work of Blum on the Medial Axis Transform (MAT) [1819].

\section{Axial Lines as Ridges on Isovist Fields}

An isovist is the space defined around a point (or centroid) from which an object can move in any direction before it encounters some obstacle. We shall see that the paradigm shift from the set of maximal discs inside the object (as in the MAT) to the maximal straight line that can be fit inside its isovists holds a key to understanding what axial lines are.

As in 'space syntax', we simplify the problem by eliminating terrain elevation and associate each isovist centroid with a pair of horizontal coordinates $(x, y)$ and a third coordinate - the length of the longest straight line across the isovist at each point which we define on the lattice as $\Delta_{i, j}^{\max }$. We extend previous work by Rana [3], where he noted that "the ridge lines give an indication of the disposition of the axial lines", by using a modification of the Medial Axis Transform [18 19 and the Hough Transform 20. The hypothesis states that all axial lines are ridges on the surface of $\Delta_{i, j}^{\max }$. The reader can absorb the concept by 'embodying' herself in the $\Delta_{i, j}^{\max }$ landscape: movement along the perpendicular direction to an axial line implies a decrease along the $\Delta_{i, j}^{\max }$ surface; and $\Delta_{i, j}^{\max }$ is an invariant, both along the axial line and along the ridge. The hypothesis goes further to predict that the converse is also true, i.e., that up to an issue of scale, all ridges on the $\Delta_{i, j}^{\max }$ landscape are axial lines.

Here we sample isovist fields by generating isovists for the set of points on a regular lattice $221 \mid 822$. Specifically, we are interested in the isovist field defined by the length of the longest straight line across the isovist at each mesh point, $(i, j)$. This measure is denoted the maximum diametric length, $\Delta_{i, j}^{\max }$ [4], or the maximum of the sum of the length of the lines of sight in two opposite directions [8, p 204]. To simplify notation, we will prefer the former term.

First, we generate a Digital Elevation Model (DEM) 23] of the isovist field, where $\Delta_{i, j}^{\max }$ is associated with mesh point $(i, j)$ [218. Our algorithm detects ridges by extracting the strict maxima (i.e. a cell with value stricly greater than any of its nearest neighbours [24]) of the discrete DEM. Next, we use an image processing transformation (the Hough Transform) on a binary image containing the local maxima points which lets us rank the detected lines in the Hough parameter space. Finally, we invert the Hough transform to find the location of axial lines on the original image.

The process of using the HT to detect lines in an image involves the computation of the HT for the entire image, accumulating evidence in an array for 
a)

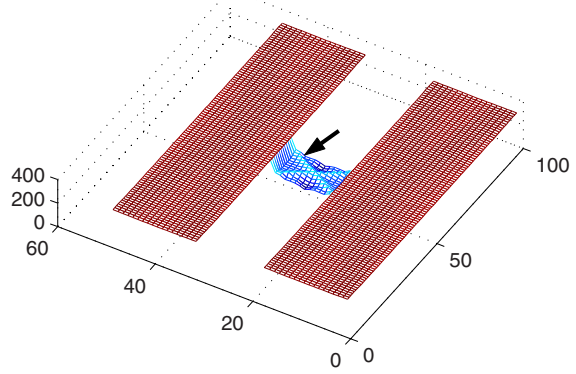

b)

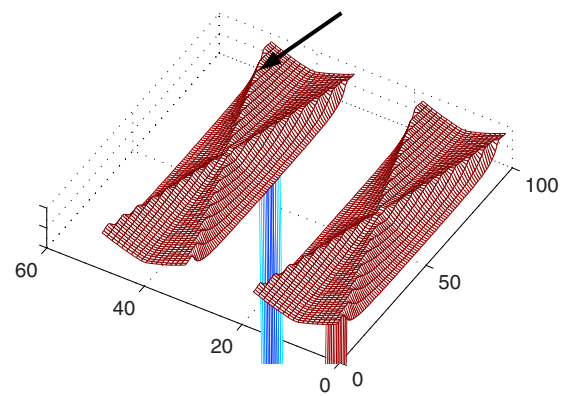

Fig. 1. (a) Plot of the Maximum Diametric Length $\left(\Delta_{i, j}^{\max }\right)$ isovist field for an ' $H$ ' shapped open space structure. (b) Zoom-in (detail) of (a) showing the ridges on the longer arms of the 'H' shape. Arrows point to the ridges o nboth figures.

events by a voting (counting) scheme and searching the accumulator array for peaks which hold information of potential lines present in the input image. The peaks provide only the length of the normal to the line and the angle that the normal makes with the $y$-axis. They do not provide any information regarding the length, position or end points of the line segment in the image plane [25]. Our line detection algorithm starts by extracting the point that has the largest number of votes on parameter space, which corresponds to the line defined by the largest number of collinear local maxima of $\Delta_{i, j}^{\max }$, and proceeds by extracting lines in rank order of the number of their votes on parameter space. One of us [4] has previously proposed rank-order methods as a rigorous formulation of the procedure originally outlined of "first finding the longest straight line that can be drawn, then the second longest line and so on (...)" [1, p 99].

To test the hypothesis that axial lines are equivalent to ridges on the $\Delta_{i, j}^{\max }$ surface, we start with a simple geometric example: an ' $\mathrm{H}$ ' shaped open space structure. As illustrated in Fig. 1, axial lines are equivalent to ridges for this simple geometric example, if extended until the borders on the open space. Indeed, one confirms this both in Fig. 17a) and Fig. 10) by properly zooming-in the $\Delta_{i, j}^{\max }$ landscape. Next, we aim at developing a method to extract these ridges as lines by sampling. In Fig. $2 \mathrm{a}$ ), we plot the local maxima of the discretized $\Delta_{i, j}^{\max }$ landscape, which are a discretized signature of the ridges on the $\Delta_{i, j}^{\max }$ continuous field. Figure [2]) is the Hough transform of Fig. 2 a) where $\theta$ goes from $0^{\circ}$ to $180^{\circ}$ in increments of $1^{\circ}$. The peaks on Fig. 2 $\mathrm{b}$ ) are the maxima in parameter space, $(\rho, \theta)$, which are ranked by height in Fig. $2 \mathrm{c})$. Finally, the ranked maxima in parameter space are inverted onto the coordinates of the lines in the original space, yielding the detected lines which are plotted on Fig. 23).

Having tested the hypothesis on a simple geometry, we repeat the procedure for the French town of Gassin - see Fig. 3. We have scanned the open space structure of Gassin [1, p 91] as a binary image and reduced the resolution of the scanned image to $300 \mathrm{dpi}$ (see inset of Fig. 3). The resulting image has $171 \times 300$ points, and is read into a Matlab matrix. 

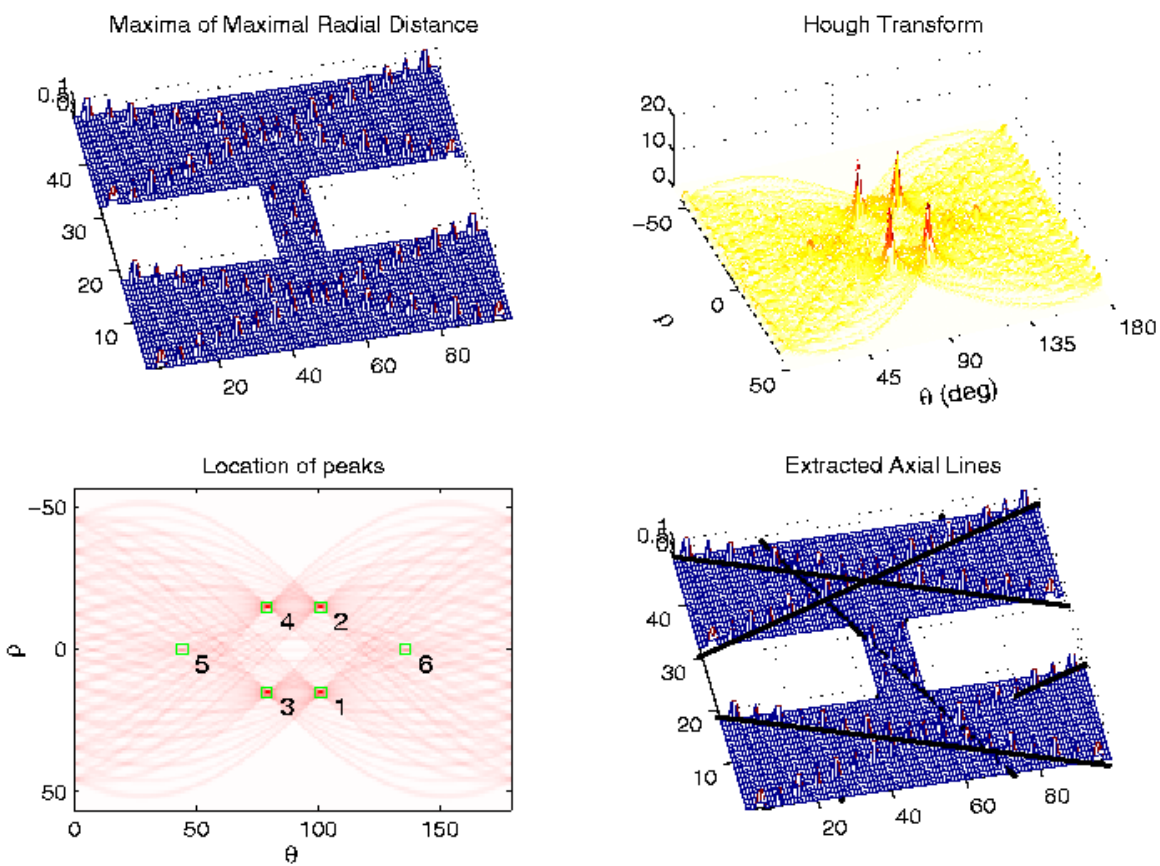

Fig. 2. (a) Local maxima of the Maximum Diametric Length $\left(\Delta_{i, j}^{\max }\right)$ for the 'H' shaped structure in Fig. 1. (b) Hough transform of (a). (c) rank of the local maxima of the surface in (b). (d) The Hough transform is inverted and the 6 highest peaks in (c) define the axial lines shown.

Next we use a ray-tracing algorithm in Matlab (angle step $=0.01^{\circ}$ ) to determine the $\Delta_{i, j}^{\max }$ measure for each point in the mesh that corresponds to open space. The landscape of $\Delta_{i, j}^{\max }$ is plot on Fig. 3. To extract the ridges on this landscape, we determine the local maxima. Next, we apply the Hough Transform, as in the ' $H$ ' shape example, and invert it to determine the 6 first axial lines (see Fig. 4). We should alert readers to the fact that as we have not imposed any boundary conditions on our definition of lines from the Hough Transform, three of these lines intersect building forms illustrating that the technique is identifying the dominant linear features in image space but ignoring any obstacles which interfere with the continuity of these linear features. We consider that this is a detail that can be addressed in subsequent development of the approach.

\section{Where Do We Go from Here?}

Most axial representations of images aim at a simplified representation of the original image, in graph form and without the loss of morphological information. Therefore, most shape graphs are invertible -a characteristic not shared with axial maps, as the original shape cannot be uniquely reconstructed from the 


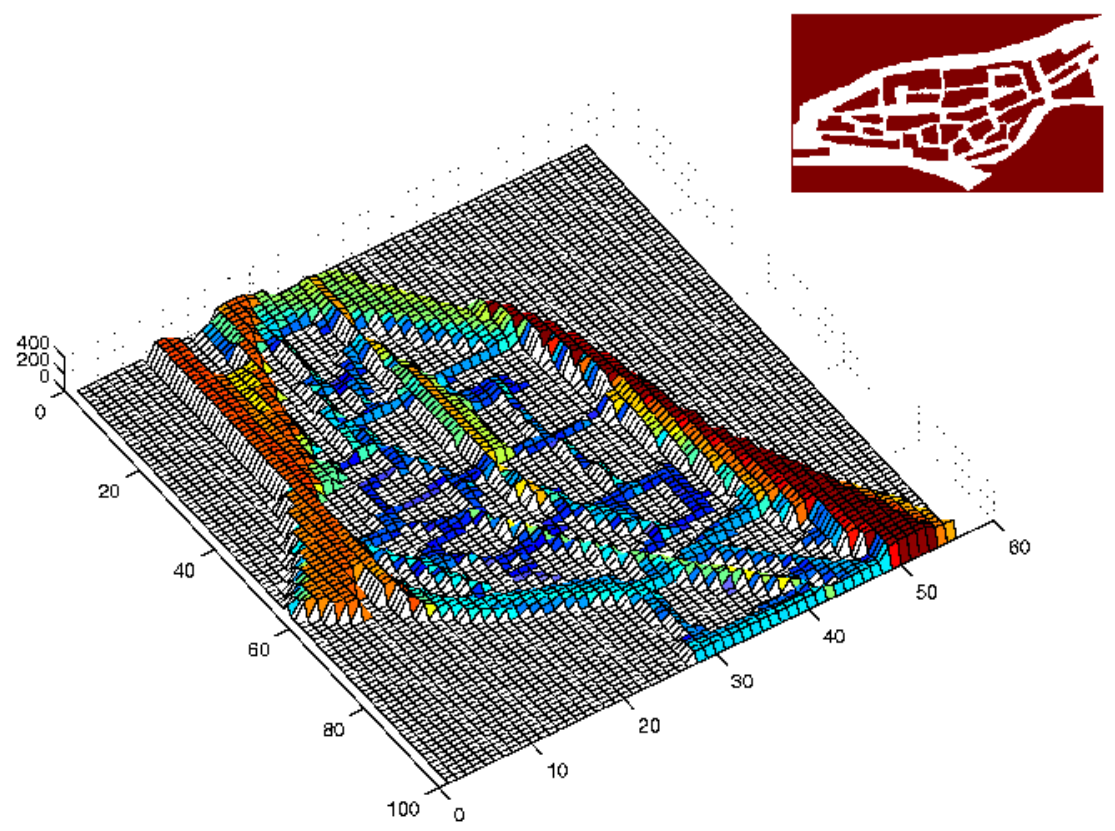

Fig. 3. Plot of the Maximum Diametric Length $\left(\Delta_{i, j}^{\max }\right)$ isovist field for the town of Gassin. The inset shows the scanned image from [1]

latter. Also, metric information on the nodes length is often stored together with the nodes (the latter often being weighted by the former), whereas it is discharged in axial maps. On the other hand, most skeletonizations aim at a representation of shape as the human observer sees it and therefore aim mostly at small scale shapes (images), whereas the process of generating axial maps assumes that the observer is immersed in the shape and aims at the representation of large scale shapes (environments). Nevertheless, we have shown that the extraction of axial lines can be accomplished with methods very similar to those routinely employed in pattern recognition and computer vision (e.g. the Medial Axial Transform and the Hough Transform).

The hypothesis has successfully passed the test of extracting axial lines both for a simple geometry and for a classical case study in Space Syntax - the town of Gassin. Indeed, $l_{2, \text { detected }}, l_{3, \text { detected }}, l_{4, \text { detected }}, l_{5, \text { detected }}$ and $l_{6, \text { detected }}$ in Fig. (4) all match reasonably well lines originally drawn [1]. Differences between original and detected lines appear for $l_{3, \text { original }}$ and $l_{3, \text { detected }}$, where the mesh we used to detect lines was not fine enough to account for the detail of the geometry and the HT counts collinear points along a line that intersects buildings, and for $l_{5, \text { original }}$ and $l_{5, \text { detected }}$, where the original solution is clearly not the longest line through the space.

Fig. 4 highlights two fundamental issues. First, defining axial lines as the longest lines of sight may lead to unconnected lines on the urban periphery. The 

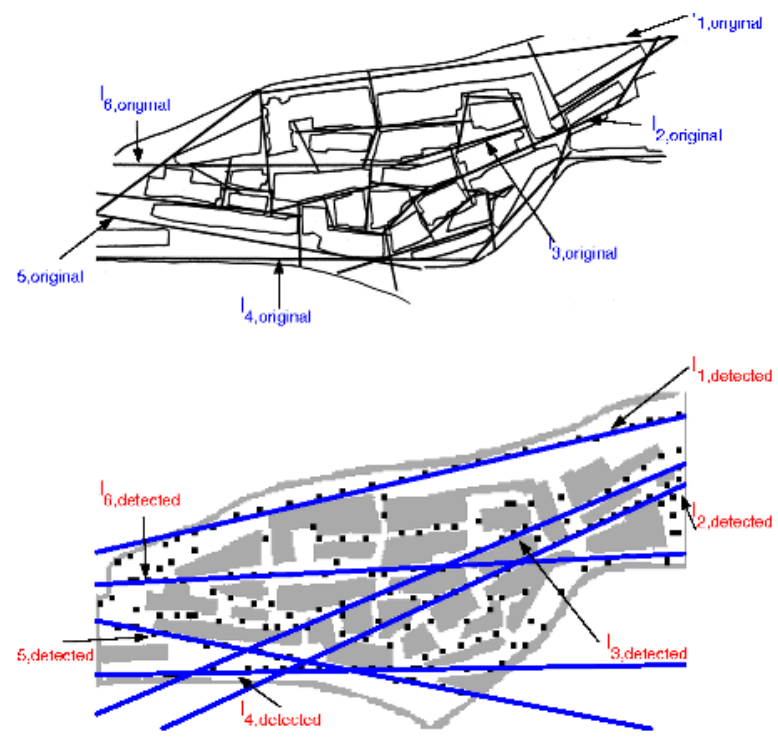

Fig. 4. (a) Axial lines for the town of Gassin [1]. (b) Local maxima of $\Delta_{i, j}^{\max }$ (squares) and lines detected by the proposed algorithm.

problem is quite evident with line $l_{1 \text {, original }}$ in Fig. 44) [1] p 91], where the solution to the longest line crossing the space is $l_{1 \text {, detected }}-$ see Fig. $4 \mathrm{~b}$ ). Thus, the price to pay for a rigorous algorithm may be that not all expected connections are traced. The second problem is an issue of scale, as one could continue identifying more local ridges with increasing image resolution (see discussion in 4]). We believe that the problem is solved if the width of the narrowest street is selected as a threshold for the length of axial lines detected from ridges on isovist fields. Only lines with length higher than the threshold are extracted. We speculate that this satisfies almost always the condition that all possible links are established, but are aware that more lines may be extracted automatically than by humanprocessing. Again, this seems to be the price to pay for a rigorous algorithm.

By being purely local, our method gives a solution to the global problem of tracing axial maps in a time proportional to the number of mesh points. Thus, algorithm optimization is akin to local optimization (mesh placement and raytracing algorithm). Although most of the present effort has been in testing the hypothesis, it is obvious that regular grids are largely redundant. Indeed, much optimization could be accomplished by generating denser grids near points where the derivative of the boundary is away from zero (e.g. turns) to improve detection at the extremities of axial lines. Also, the algorithm could be improved by generating iterative solutions that would increase grid and angle sweep resolutions until a satisfactory solution would be reached or by parallelizing visibility analysis calculations [26].

Our approach to axial map extraction is preliminary as the HT detects only line parameters while axial lines are line segments. Nevertheless, there has been 
considerable research effort put into line segment detection in urban systems, generated mainly by the detection of road lane markers [27 28], and we are confident that further improvements involve only existing theory.

This note shows that global entities in urban morphology can be defined with a purely local approach. We have shown that there is no need to invoke the concept of convex space to define axial lines. By providing rigorous algorithms inspired by work in pattern recognition and computer vision, we have started to uncover problems implicit in the original definition (disconnected lines at boundary, scale issues), but have proposed working solutions to all of them which, we believe will engage other disciplines in the effort of gaining insight into urban morphology. Finally, we look with considerable optimism to the automatic extraction of axial lines and axial maps in the near future and believe that automatic processing of medium to large scale cities may be only a few years away from being implemented on desktop computers.

Acknowledgments. RC acknowledges generous financial support from Grant EPSRC GR/N21376/01 and is grateful to Bill Hillier and Alan Penn for valuable comments. The authors are indebted to Sanjay Rana both for the original idea that axial lines appear as ridges in the MDL isovist field and for using his Isovist Analyst Extension (see http://www.geog.le.ac.uk/ sanjayrana/software_isovistanalyst.htm) to provide independent corroboration on the ' $\mathrm{H}$ ' test problem.

\section{References}

1. Hillier, B., Hanson, J.: The Social Logic of Space. Cambridge University Press, Cambridge (1984)

2. Benedikt, M.: To take hold of space: isovists and isovist fields. Environ. Plan. B 6 (1979) 47-65

3. Rana, S.: Isovist analyst extension for arcview 3.2 (2002)

4. Batty, M., Rana, S.: The automatic definition and generation of axial lines and axial maps. Environ. Plan. B 31 (2004) forthcoming.

5. Hillier, B., Penn, A., Hanson, J., T, T.G., Xu, J.: Natural movement: or, configuration and attraction in urban pedestrian movement. Environ. Plan. B 20 (1993) 29-66

6. Peponis, J., Ross, C., Rashid, M.: The structure of urban space, movement and co-presence: The case of atlanta. Geoforum 28 (1997) 341-358

7. Peponis, J., Wineman, J., Bafna, S., Rashid, M., Kim, S.: On the generation of linear representations of spatial configuration. Environ. Plan. B 25 (1998) 559-576

8. Ratti, C.: Urban analysis for environmental prediction. Phd thesis, University of Cambridge (2001)

9. Albert, R., Barabási, A.L.: Statistical mechanics of complex networks. Rev. Mod. Phys. 74 (2002) 47-97

10. Newman, M.E.J.: The structure and function of complex networks. SIAM Rev. 45 (2003) 167-256

11. Batty, M., Longley, P.: Fractal Cities. Academic Press, San Diego, CA (1994) 
12. Carvalho, R., Penn, A.: Scaling and universality in the micro-structure of urban space. Physica A 332 (2004) 539-547

13. Frankhauser, P.: La Fractalité des Structures Urbaines. Anthropos, Paris (1994)

14. Makse, H.A., Havlin, S., Stanley, H.E.: Modelling urban growth patterns. Nature 377 (1995) 608-612

15. Makse, H.A., Jr, J.S.A., Batty, M., Havlin, S., Stanley, H.E.: Modeling urban growth patterns with correlated percolation. Phys. Rev. E 58 (1998) 7054-7062

16. Latora, V., Marchiori, M.: Is the boston subway a small-world network? Physica A 314 (2002) 109-113

17. Chowell, G., Hyman, J.M., Eubank, S., Castillo-Chavez, C.: Scaling laws for the movement of people between locations in a large city. Phys. Rev. E 68 (2003) 066102

18. Blum, H.: Biological shape and visual science (part 1). J. Theor. Biol. 38 (1973) 205-287

19. Blum, H., Nagel, R.N.: Shape description using weighted symmetric features. Pattern Recogn. 10 (1978) 167-180

20. Illingworth, J., Kittler, J.: A survey of the hough transform. Computer Vision, Graphics, and Image Processing 44 (1988) 87-116

21. Batty, M.: Exploring isovist fields: space and shape in architectural and urban morphology. Environ. Plan. B 28 (2001) 123-150

22. Turner, A., Doxa, M., O'Sullivan, D., Penn, A.: From isovists to visibility graphs: a methodology for the analysis of architectural space. Environ. Plan. B 28 (2001) 103-121

23. Burrough, P.A., McDonnell, R.A.: Principles of Geographical Information Systems. Spatial Information Systems and Geostatistics. Oxford University Press (1998)

24. Niblack, C., Gibbons, P., Capson, D.: Generating skeletons and ceterlines from the distance transform. CVGIP: Graphical Models and Image Processing 54 (1992) 420-437

25. Gonzalez, R.C., Woods, R.E.: Digital Image Processing. Addison-Wesley (1992)

26. Mills, K., Fox, G., Heimbach, R.: Implementing an intervisibility analysis model on a parallel computing system. Computers \& Geosciences 18 (1992) 1047-1054

27. Kamat-Sadekar, V., Ganesan, S.: Complete description of multiple line segments using the hough transform. Image and Vision Computing 16 (1998) 597-613

28. Pomerleau, D., Jochem, T.: Rapidly adapting machine vision for automated vehicle steering. IEEE Expert 11 (1996) 19-27 